\title{
Detection of Parkinsonian Motor Symptoms Using Non-Invasive Motion Sensing Technology
}

\author{
John Sakaleros ${ }^{1}$, Farzin Shamloo ${ }^{2}$, Aditya Shanghavi ${ }^{3}$, Anne Sereno ${ }^{1,2,3}$ \\ ${ }^{1}$ Indiana University School of Medicine; ${ }^{2}$ Purdue University Department of Psychology; ${ }^{3}$ Purdue \\ University Department of Biomedical Engineering
}

Parkinson's Disease (PD) is characterized by impaired movement, resting tremor, and muscle rigidity. The Unified PD Rating Scale (UPDRS) is a standardized protocol used by neurologists to measure progression of disease as well as evaluation of treatments. However, this examination is subjective, time consuming, and results can be affected by stress, diet, or sleep. Our goal is to develop a non-invasive device that can record objective clinically-relevant measurements during subtasks of the UPDRS to allow for remote evaluations, which would be beneficial considering the frequency of clinical visits for medication adjustments. Five healthy individuals (ages 21-59) completed UPDRS tasks 3.6 (pronation/supination of hands) and 3.17 (rest tremor amplitude). Participants performed these tasks twice, first normally and second simulating PD patients (tremor, bradykinesia, reduction of movement amplitude) after viewing example videos. Motion data including linear and angular accelerations in 3 dimensions was acquired using a lightweight wrist-mounted motion sensor. Three features were extracted: (1) Power of higher frequency components of the linear acceleration signal (rest task), as a measure of resting tremor amplitude. (2) Power of higher frequency components of the rotational acceleration signal (pronation/supination task), as a measure of bradykinesia. (3) Standard deviation of the local maxima of the rotational acceleration (pronation/supination task), as a measure of reduction in movement speed and amplitude. These features were used to correctly differentiate trials completed with and without simulated PD symptoms, using an SVM classifier with leave-one-out cross validation accuracy of $95 \%$. These findings suggest it is possible to capture clinical features of PD using motion sensors. Future work in PD patients will examine how these measures correlate with UPDRS evaluations and whether they will be helpful in providing a quick, objective telehealth measure of progression and treatment response that can supplement current tools. 